\title{
Median nodule of the upper lip
}

INSERM

\section{Source}

INSERM. (1999). Orphanet: an online rare disease and orphan drug data base. Median nodule of the upper lip. ORPHA:2699

Median nodule of the upper lip is a minor trait of the lip transmitted in an autosomal dominant fashion. 